\title{
Dietary intake in type 1 diabetes at different stages of diabetic kidney disease
}

\author{
Aila J. Ahola ${ }^{a, b, c}$, Carol Forsblom ${ }^{a, b, c}$, Valma Harjutsalo ${ }^{a, b, c, d}$, Per-Henrik Groop ${ }^{a, b, c, e, *}$ \\ ${ }^{a}$ Folkhälsan Institute of Genetics, Folkhälsan Research Center, Helsinki, Finland \\ ${ }^{\mathrm{b}}$ Abdominal Center Nephrology, University of Helsinki and Helsinki University Central Hospital, Helsinki, Finland \\ ${ }^{\mathrm{c}}$ Research Program for Clinical and Molecular Metabolism, Faculty of Medicine, University of Helsinki, Finland \\ d Diabetes Prevention Unit, National Institute for Health and Welfare, Helsinki, Finland \\ e Department of Diabetes, Central Clinical School, Monash University, Melbourne, Victoria, Australia
}

\section{A R T I C L E I N F O}

\section{Article history:}

Received 15 February 2019

Received in revised form

27 May 2019

Accepted 25 June 2019

Available online 1 July 2019

\section{Keywords:}

Dietary patterns

Energy and nutrients

Food intake

Kidney function

Type 1 diabetes

\begin{abstract}
A B S T R A C T
Aim: Diet plays an important role in the kidney health of individuals with type 1 diabetes. However, not much is known about dietary practices at different stages of diabetic nephropathy. We aimed at investigating food intake, dietary patterns, and nutrient intakes in individuals with type 1 diabetes differing in renal status.

Methods: Data were available from 1874 individuals with type 1 diabetes (45\% men, age 48 \pm 13 years). Diet was assessed at the levels of food items and diet patterns (diet questionnaire), and energy and nutrient intakes (food record). Six groups were formed based on the eGFR or dialysis and transplantation status.

Results: Reductions in liquid-milk product and salt consumption, and increase in special diet adherence were observed at the early stages of eGFR decline. Reduced coffee consumption was observed after eGFR was $<30 \mathrm{ml} / \mathrm{min} / 1.73 \mathrm{~m}^{2}$. With advancing kidney failure, rye bread consumption decreased, but that of wheat bread increased. Compared to those with intact kidney function (the index group), the Fish and vegetable diet pattern scores were higher in individuals with mildly-to-severely decreased eGFR. Instead, the Sweet pattern scores were lower than in the index group in all other groups. Energy intake was lower in all groups compared to those with intact kidney function. Advancing kidney failure was associated with reductions in protein intake per body weight, and in the intakes of sodium, potassium, calcium, and phosphorus.

Conclusions: Differences in the dietary intake are seen already at the early stages of kidney function decline.
\end{abstract}

(c) 2019 Published by Elsevier B.V.

\section{Introduction}

Increasing prevalence of chronic kidney disease (CKD), high costs of treating individuals with kidney failure, and poor outcomes related to renal replacement therapies are important health challenges across the globe [1]. It has been suggested, that the overall burden of CKD is largely driven by the increase in the prevalence of diabetes, mainly that of type 2

\footnotetext{
* Corresponding author at: Folkhälsan Research Center, Biomedicum Helsinki C318b, PO Box 63, FI-00014 University of Helsinki, Finland (P.-H. Groop).

E-mail address: per-henrik.groop@helsinki.fi (P.-H. Groop).
} 
[2]. However CKD is a major health concern also in type 1 diabetes (T1D) as, over the course of a lifetime, diabetic nephropathy affects approximately one third of this population [3]. Importantly, the presence of diabetic nephropathy greatly increases the risk of premature death of patients with T1D [4].

Targeting the blood glucose and blood pressure levels near those physiological are among the major approaches for minimizing the risk of diabetes complications. In addition modifiable life-style factors, such as diet, may play an important role in the progression and management of diabetic kidney disease [5]. With respect to food intake, overall healthy diet, and reduced intakes of sodium and protein have been commonly acknowledged $[6,7]$.

For the prevention of diabetic kidney disease no particular dietary recommendation exist, however. Instead, the current recommendations stress the need for individualized meal plan; one that, amongst others, takes into consideration the patient's age, size, metabolic goals, and stage of kidney disease $[8,9]$. As with deteriorating renal function the kidneys' ability to remove waste products and extra water from the blood gets compromised, phosphorus, potassium, and fluid restrictions are typically issued in the pre-dialysis period [10]. These dietary restrictions may lead to limited intakes of fruits, vegetables, grains, and nuts, and therefore impair the overall quality of the diet [11]. In contrast, the advent of dialysis poses new challenges in the form of protein loss in the dialysate and increased risk of anorexia due to uraemia, nausea, and loss of appetite, which again needs to be reflected in the diet plan $[10,12]$.

While a number of studies have been published about dietary intake and the risk of diabetic kidney disease, the studies describing the actual diets of individuals with T1D at different stages of renal disease are practically non-existent. In this study, we wanted to fill in this gap in knowledge and describe the diets of the Finnish Diabetic Nephropathy (FinnDiane) Study participants divided by stages of kidney disease. To get an extensive view, the dietary intake was investigated at the levels of food consumption, dietary patterns, and nutrients.

\section{Materials and methods}

\subsection{Subjects}

All individuals with T1D participating in the FinnDiane Study were included in this cross-sectional observational study if they had known renal status and had completed a diet questionnaire within a year from the renal assessment. T1D was defined as diabetes onset before the age of 35 years, and permanent insulin treatment, initiated within a year from the diagnosis. The Ethics Committee of The Helsinki and Uusimaa Hospital District approved the study protocol. Written informed consent was obtained from all participants.

\subsection{Clinical and laboratory data}

At the study visit, all participants were thoroughly investigated [13]. This included measurements of body weight, height, blood pressure, and waist and hip circumference. Body mass index (BMI; $\mathrm{kg} / \mathrm{m}^{2}$ ) was calculated and participants were categorized into those underweight $\left(\mathrm{BMI}<18.5 \mathrm{~kg} / \mathrm{m}^{2}\right)$, normal-weight (BMI $18.5-<25 \mathrm{~kg} / \mathrm{m}^{2}$ ), overweight (BMI $\geq 25-$ $\left.<30 \mathrm{~kg} / \mathrm{m}^{2}\right)$, and obese $\left(\geq 30 \mathrm{~kg} / \mathrm{m}^{2}\right)$. Waist-to-hip ratio, and the mean of two blood pressure measurements were calculated. $\mathrm{HbA}_{1 \mathrm{c}}$ was measured locally using a standardized assay. Blood was drawn for subsequent central analyses of serum lipids, lipoproteins, and creatinine concentrations. Fasting was not required, and a light breakfast was allowed to prevent or treat hypoglycaemia. Serum creatinine concentration was used to calculate the estimated glomerular filtration rate (eGFR as $\mathrm{ml} / \mathrm{min} / 1.73 \mathrm{~m}^{2}$ ) as described by Levey et al [14]. Using a standardized form, the attending physician recorded the participant's medication use and diabetes complications. From these records and medical files, we obtained data on dialysis and kidney transplantation. Following classifications were made; normal or high eGFR (eGFR ${ }^{\geq 90}$ ), mildly decreased eGFR (eGFR ${ }^{\geq 60-89}$ ), mildly to severely decreased eGFR $\left(\right.$ eGFR ${ }^{\geq 30-59}$ ), severely decreased eGFR (eGFR ${ }^{<30}$ ), dialysis, and kidney transplantation. Classifications based on eGFR are modified from the KDIGO 2012 clinical practice guidelines for the evaluation and management of CKD [15]. Smoking was self-reported.

\subsection{Dietary intake}

Two methods to study dietary intake were used as previously described [16]. In short, participants first completed a validated diet questionnaire [17]. Using this questionnaire, our aim was to obtain an overall picture of the participants' dietary practices. Dietary habits related to coffee, tea, liquid milk product, bread, spread, cooking fat, and salt consumption were queried. Participants reported the use of probiotic products and dietary supplements, and whether they adhered to any special diets or had received dietary counselling from health-care professionals. On a five-level scale, the level of adherence with these recommendations was estimated. Individuals self-reportedly following the recommendations always or most of the time were considered as "compliant". Included was also a 19-item food frequency questionnaire (FFQ) where consumption frequencies of fish dishes, meat dishes, poultry, sausages and cold-cuts, eggs, legumes, fresh vegetables, cooked vegetables, potatos, pasta and rice, fruits and berries, full-fat cheese, low-fat cheese, yoghurt and curd, ice cream, soft drinks, sweet pastries, sweets, and fried and grilled foods were reported using a seven-level response scale (several times per day, once a day, 4-6 times per week, 2-3 times per week, once a week, 1-3 times per month, and less frequently or never). Upon returning the diet questionnaire, participants were sent an allocated 3-day diet record covering two weekdays and one weekend day. Another 3-day record was completed within 2-3 months. Mean dietary intake was calculated based on the 6-day food record for those completing both records, and the 3-day food record for those completing only the first recording. Detailed instructions for completing the record were provided. AivoDiet software (version 2.0.2.3, AIVO, Turku, Finland) was used to calculate energy and nutrient contents. Individuals with reported 


\section{Table 1 - Participant characteristics divided by the kidney status.}

\begin{tabular}{|c|c|c|c|c|c|c|}
\hline & $e^{e G F R}{ }^{\geq 90} n=1118$ & eGFR ${ }^{\geq 60-89} n=396$ & $e^{-G F R}{ }^{\geq 30-59} n=115$ & $e G F R^{<30} n=62$ & Dialysis $n=73$ & Transplant $\mathrm{n}=110$ \\
\hline Men, \% & 44.7 & $37.1^{b}$ & 48.7 & 41.9 & $58.9^{a}$ & $56.4^{\mathrm{a}}$ \\
\hline Current smoker, \% & 15.6 & $11.4^{\mathrm{a}}$ & 16.7 & 11.7 & 14.3 & 15.4 \\
\hline Age, years & $43(35,53)$ & $58(49,65)^{c}$ & $59(50,64)^{c}$ & $54(47,62)^{c}$ & $49(43,53)^{c}$ & $52(46,60)^{c}$ \\
\hline Diabetes duration, years & $24(18,34)$ & $38(28,47)^{c}$ & $42(34,49)^{\mathrm{c}}$ & $39(30,46)^{\mathrm{c}}$ & $37(29,43)^{c}$ & $40(35,44)^{c}$ \\
\hline $\mathrm{SBP}, \mathrm{mmHg}$ & $133(122,144)$ & $139(127,156)^{c}$ & $148(134,163)^{\mathrm{c}}$ & $143(135,157)^{\mathrm{c}}$ & $145(132,162)^{c}$ & $153(137,167)^{c}$ \\
\hline DBP, $\mathrm{mmHg}$ & $79(72,85)$ & $75(69,81)^{\mathrm{c}}$ & $77(69,83)^{\mathrm{a}}$ & $80(68,87)$ & $79(71,88)$ & $76(70,86)$ \\
\hline BMI, $\mathrm{kg} / \mathrm{m}^{2}$ & $26(23,28)$ & $26(23,28)$ & $27(23,30)^{b}$ & $25(23,28)$ & $24(22,27)^{b}$ & $24(21,27)^{c}$ \\
\hline Underweight, \% & 1.3 & 0.3 & $0.0^{\mathrm{b}}$ & 3.3 & $2.9^{\mathrm{a}}$ & $2.8^{\mathrm{a}}$ \\
\hline Normal-weight, \% & 43.7 & 39.0 & 31.0 & 42.6 & 60.0 & 56.0 \\
\hline Overweight, \% & 40.1 & 44.9 & 43.4 & 36.1 & 30.0 & 28.4 \\
\hline Obese, $\%$ & 15.0 & 15.8 & 25.7 & 18.0 & 7.1 & 12.8 \\
\hline Waist circumference, $\mathrm{cm}$ & $87(79,96)$ & $88(79,97)$ & $97(82,107)^{c}$ & $85(78,101)$ & $89(84,100)$ & $88(79,98)$ \\
\hline Hip circumference, $\mathrm{cm}$ & $99(94,106)$ & $100(95,106)$ & $101(95,110)^{\mathrm{a}}$ & $99(94,107)$ & $97(92,102)^{\mathrm{a}}$ & $97(92,102)^{\mathrm{b}}$ \\
\hline Waist-to-hip ratio & $0.87(0.82,0.93)$ & $0.88(0.81,0.94)$ & $0.92(0.83,1.00)^{c}$ & $0.88(0.81,0.97)$ & $0.94(0.88,1.00)^{\mathrm{c}}$ & $0.90(0.85,0.98)^{c}$ \\
\hline $\mathrm{HbA}_{1 \mathrm{c}}, \mathrm{mmol} / \mathrm{mol}$ & $65(57,74)$ & $64(55,73)$ & $66(59,75)$ & $67(56,77)$ & $62(53,73)$ & $64(53,75)$ \\
\hline $\mathrm{HbA}_{1 \mathrm{c}}, \%$ & $8.1(7.4,8.9)$ & $8.0(7.2,8.8)$ & $8.2(7.5,9.0)$ & $8.3(7.3,9.2)$ & $7.8(7.0,8.8)$ & $8.0(7.0,9.0)$ \\
\hline Triglycerides, mmol/l & $0.9(0.7,1.3)$ & $1.0(0.7,1.2)$ & $1.2(0.9,1.7)^{\mathrm{c}}$ & $1.4(0.9,2.0)^{\mathrm{c}}$ & $1.2(1.0,1.7)^{\mathrm{c}}$ & $1.3(0.9,1.6)^{c}$ \\
\hline Total cholesterol, mmol/l & $4.5(4.0,5.1)$ & $4.6(4.0,5.1)$ & $4.4(3.9,4.9)^{\mathrm{a}}$ & $4.0(3.4,4.7)^{c}$ & $3.8(3.2,4.4)^{c}$ & $4.2(3.6,4.8)^{c}$ \\
\hline HDL cholesterol, $\mathrm{mmol} / \mathrm{l}$ & $1.6(1.3,1.9)$ & $1.7(1.4,2.0)^{c}$ & $1.5(1.2,1.8)$ & $1.4(1.1,1.7)^{b}$ & $1.3(1.1,1.6)^{c}$ & $1.4(1.2,1.7)^{b}$ \\
\hline Lipid-lowering medication, \% & 26.7 & $46.2^{\mathrm{C}}$ & $56.5^{c}$ & $58.3^{\mathrm{C}}$ & $72.5^{\mathrm{c}}$ & $66.0^{\mathrm{C}}$ \\
\hline Antihypertensive medication, \% & 35.9 & $66.0^{c}$ & $94.5^{c}$ & $100^{c}$ & $90.0^{c}$ & $94.3^{\mathrm{c}}$ \\
\hline Vascular hard event, \% & 6.1 & $19.6^{\mathrm{c}}$ & $36.6^{c}$ & $38.7^{c}$ & $44.4^{c}$ & $41.8^{\mathrm{c}}$ \\
\hline Proliferative retinopathy, \% & 22.0 & $42.6^{c}$ & $79.8^{c}$ & $88.5^{c}$ & $80.8^{c}$ & $94.5^{c}$ \\
\hline \multicolumn{7}{|c|}{$\begin{array}{l}\text { Data are presented as frequencies or median (interquartile range). In these variables, comparisons between the highest eGFR group and the other groups were done with Chi-squared test and Mann- } \\
\text { Whitney } U \text { test, respectively. eGFR, estimated glomerular filtration rate (unit } \mathrm{ml} / \mathrm{min} / 1.73 \mathrm{~m}^{2} \text { ); SBP, systolic blood pressure; DBP, diastolic blood pressure; BMI, body-mass index; underweight, } \\
\text { BMI }<18.5 \mathrm{~kg} / \mathrm{m}^{2} \text {; normal-weight, BMI } 18.5-<25.0 \mathrm{~kg} / \mathrm{m}^{2} \text {; overweight, BMI } \geq 25.0-<30 \mathrm{~kg} / \mathrm{m}^{2} \text {; obese, BMI } \geq 30 \mathrm{~kg} / \mathrm{m}^{2} \text {; vascular hard event, acute myocardial infarction, coronary bypass, stroke, } \\
\text { amputation, or peripheral vascular disease. } \\
\text { a } p<0.05 \text {. } \\
\text { b } p<0.01 \text {. } \\
\text { c } p<0.001 .\end{array}$} \\
\hline
\end{tabular}




\section{Table 2 - Food consumption, compliance, and use of probiotics and dietary supplements divided by the renal status.}

\begin{tabular}{|c|c|c|c|c|c|c|}
\hline & $e G F R^{\geq 90} n=1118$ & eGFR ${ }^{\geq 60-89} n=396$ & $e G^{2}{ }^{230-59} n=115$ & $\mathrm{eGFR}^{<30} \mathrm{n}=62$ & Dialysis $n=73$ & Transplant $\mathrm{n}=110$ \\
\hline Coffee, cups per day & $3.5(2.0,5.0)$ & $4.0(2.0,5.5)$ & $3.5(2.0,4.5)$ & $2.3(1.0,4.0)^{b}$ & $2.5(1.5,3.0)^{c}$ & $3.0(2.0,4.0)^{\mathrm{b}}$ \\
\hline Tea, cups per day & $0(0,1)$ & $0(0,1)$ & $0(0,1.5)^{b}$ & $0(0,2)^{a}$ & $0(0,1)$ & $0(0,2)^{b}$ \\
\hline Liquid milk products, glasses per day & $2.5(1.0,4.0)$ & $2.0(1.0,3.0)^{c}$ & $2.0(0.5,3.0)^{b}$ & $1.0(0,2.0)^{c}$ & $1.0(0,2.0)^{c}$ & $2.0(1.0,3.0)^{\mathrm{a}}$ \\
\hline Bread, slices per day & $4.5 \pm 2.3$ & $4.5 \pm 2.1$ & $4.5 \pm 1.9$ & $4.3 \pm 2.0$ & $4.1 \pm 2.8^{\mathrm{a}}$ & $4.7 \pm 2.3$ \\
\hline Rye bread, \% & 85.4 & 83.5 & 80.9 & $35.5^{c}$ & $27.8^{\mathrm{C}}$ & 81.5 \\
\hline Wheat bread, \% & 10.5 & 8.6 & 14.8 & $50.0^{c}$ & $65.3^{c}$ & 14.8 \\
\hline Vegetable oil-based spread, \% & 61.1 & 60.3 & 67.8 & 61.3 & 71.2 & $72.6^{\mathrm{a}}$ \\
\hline Vegetable oil-based cooking fats, \% & 79.9 & $74.7^{\mathrm{a}}$ & 77.4 & 71.0 & $68.5^{\mathrm{a}}$ & 77.8 \\
\hline Aims at reducing salt consumption, $\%$ & 20.8 & $31.1^{\mathrm{c}}$ & $34.8^{\mathrm{b}}$ & $39.3^{\mathrm{b}}$ & $38.9^{b}$ & $30.8^{\mathrm{a}}$ \\
\hline Probiotics, \% & 32.9 & 33.0 & 30.7 & 22.6 & 34.2 & 28.4 \\
\hline Dietary supplements, \% & 60.3 & 60.5 & $50.4^{\mathrm{a}}$ & $43.5^{\mathrm{a}}$ & 52.1 & $43.1^{\mathrm{b}}$ \\
\hline \multicolumn{7}{|l|}{ Has received dietary counselling from } \\
\hline Dietitian, \% & 56.5 & 60.0 & $71.3^{\mathrm{b}}$ & $83.9^{c}$ & $84.9^{c}$ & $76.6^{c}$ \\
\hline Nurse, \% & 52.6 & 54.9 & 56.5 & 41.9 & $38.4^{\mathrm{a}}$ & 55.1 \\
\hline Physician, \% & 20.0 & 21.8 & 21.7 & $33.9^{\mathrm{a}}$ & 30.1 & 25.2 \\
\hline Compliant, \% & 47.6 & $56.2^{\mathrm{b}}$ & $61.1^{\mathrm{b}}$ & $85.2^{\mathrm{C}}$ & $75.3^{c}$ & $69.4^{\mathrm{c}}$ \\
\hline Any special diet, \% & 28.7 & 33.2 & $59.1^{\mathrm{c}}$ & $86.9^{c}$ & $77.8^{c}$ & $41.9^{\mathrm{b}}$ \\
\hline \multicolumn{7}{|c|}{$\begin{array}{l}\text { Data are presented as median (interquartile range), mean } \pm \text { standard deviation, or frequency. In these variables, comparisons between the highest eGFR group and the other groups were done with } \\
\left.\text { Mann-Whitney } U \text { test, independent samples t-test, and Chi-squared test, respectively. eGFR, estimated glomerular filtration rate (unit } \mathrm{ml} / \mathrm{min} / 1.73 \mathrm{~m}{ }^{2}\right) \text {; Compliant, self-reportedly complies with the } \\
\text { dietary guidelines always or most of the time. } \\
\text { a } p<0.05 \text {. } \\
\text { b } p<0.01 \text {. } \\
\text { c } p<0.001 \text {. }\end{array}$} \\
\hline
\end{tabular}




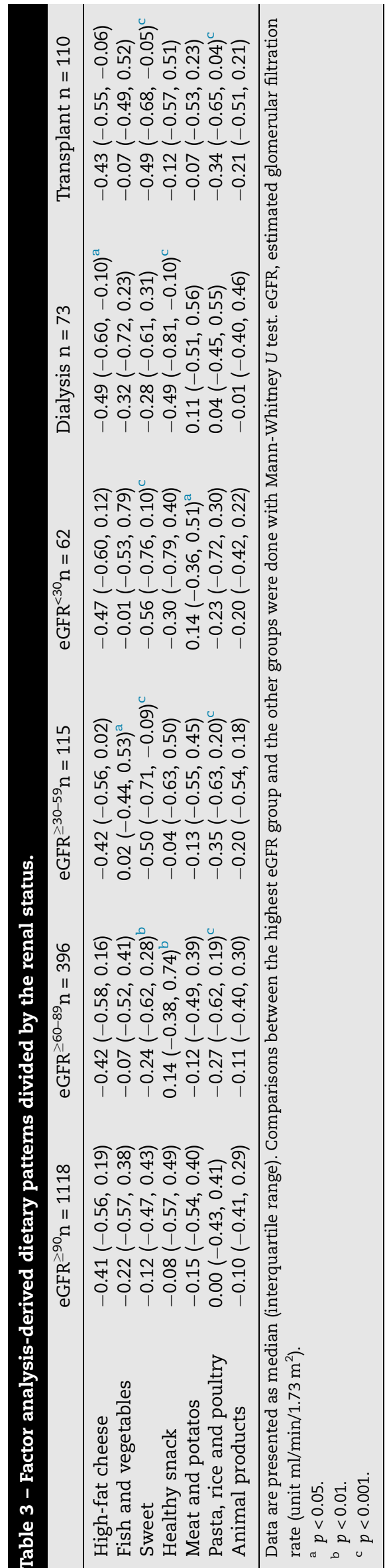

energy intake between 3349 and $14654 \mathrm{~kJ}$ (800 and $3500 \mathrm{kcal}$ ) were included in the analyses of nutrient data.

\subsection{Statistical analyses}

Entries in the FFQ were submitted to an exploratory factor analysis (maximal likelihood and varimax rotation) to yield dietary patterns. Here, we included all factors with eigenvalues $>1.0$, and in each factor we included food items with factor loadings $1 \geq 0.20$. The factor score was the sum of the scores for items associated with a particular factor multiplied by its factor loading. Categorical observations are reported as frequencies, and continuous variables are presented as means \pm standard deviations when normally distributed and as medians (interquartile ranges) when non-normally distributed. In the analyses, all other groups were compared to those with $\mathrm{eGFR}^{\geq 90}$. In addition individuals in dialysis and those with kidney transplantation were compared. For categorical variables, the between-group comparisons were conducted with Chi-squared test, and for continuous variables with independent samples' t-test and Mann-Whitney $U$ test, as appropriate. For analyses, IBM SPSS Statistics for Windows, Version 22.0 (IBM Corp, Armonk, NY, USA) was used. A twotailed $P$ value $<0.05$ denoted statistical significance.

\section{Results}

\subsection{Study participants}

Data were available from 1874 individuals (44.5\% men, age $48.1 \pm 13.4$ years). Altogether, $59.7 \%$ had eGFR $^{\geq 90}$ (index group), $21.1 \%$ had eGFR ${ }^{\geq 60-89}, 6.1 \%$ had eGFR ${ }^{\geq 30-59}, 3.3 \%$ had eGFR $^{<30}, 3.9 \%$ were on dialysis, and $5.9 \%$ were kidney transplant recipients (Table 1). Individuals on dialysis and with a kidney transplant were more frequently men compared to those with normal or high eGFR. Compared to the index group, age and diabetes duration were higher in all other groups. Individuals on dialysis and those with a kidney transplant had lower median BMI compared to those with intact kidney function. Moreover, the distribution of participants based on the four BMI classes differed significantly between these groups; notably, the frequencies of underweight and normal-weight individuals were higher, while those of overweight and obese were lower in the two advanced kidney disease groups. However, compared to the index group, the median waist-to-hip ratios were higher in the two groups with most advanced kidney disease.

\subsection{Food consumption, supplement use, and diet adherence}

The median (interquartile range) of the time gap between the dietary and renal assessment was 5 days $(0,30)$. Compared to the index group, individuals with eGFR ${ }^{<30}$, those on dialysis, and kidney transplant recipients reported consuming lower numbers of coffee cups (Table 2). Liquid milk product consumption was lower in all groups, compared to the index group. Only among individuals on dialysis was the number of bread slices consumed lower than in the index group. Dif- 
Table 4 - Energy, macronutrient, sucrose, and fibre intake divided by the renal status.

\begin{tabular}{|c|c|c|c|c|c|c|}
\hline & eGFR ${ }^{\geq 90} n=839$ & eGFR ${ }^{\geq 60-89} n=332$ & $e^{e G F R}{ }^{\geq 30-59} n=83$ & $e^{e G F R}{ }^{<30} n=38$ & Dialysis $n=36$ & Transplant $n=71$ \\
\hline Energy, MJ & $8100(6892,9479)$ & $7390(6264,8591)^{c}$ & $6794(5991,7931)^{c}$ & $6490(5616,7298)^{\mathrm{c}}$ & $6872(6070,8376)^{\mathrm{c}}$ & $6773(6066,7799)^{c}$ \\
\hline Energy, kJ/kg & $110 \pm 29$ & $101 \pm 27^{c}$ & $87 \pm 27^{c}$ & $85 \pm 22^{c}$ & $98 \pm 26^{a}$ & $99 \pm 25^{b}$ \\
\hline Carbohydrates, E\% & $42.7 \pm 7.0$ & $42.3 \pm 6.9$ & $44.3 \pm 7.0$ & $44.2 \pm 6.8$ & $42.7 \pm 5.4$ & $42.9 \pm 7.4$ \\
\hline Fats, E\% & $36.1 \pm 6.2$ & $36.0 \pm 6.5$ & $35.6 \pm 7.2$ & $37.7 \pm 6.8$ & $38.9 \pm 4.6^{a}$ & $36.3 \pm 5.8$ \\
\hline SAFA, E\% & $12.8 \pm 2.9$ & $12.6 \pm 3.0$ & $12.0 \pm 3.1^{\mathrm{a}}$ & $12.3 \pm 3.3$ & $13.9 \pm 2.9^{\mathrm{a}}$ & $12.7 \pm 2.5$ \\
\hline MUFA, E\% & $12.0(10.6,13.8)$ & $11.9(10.4,13.5)$ & $12.2(10.4,14.0)$ & $13.2(10.6,15.2)$ & $13.3(12.0,14.5)^{b}$ & $11.8(10.3,13.5)$ \\
\hline PUFA, E\% & $5.9(5.0,6.9)$ & $5.8(4.9,7.1)$ & $6.3(5.2,7.5)$ & $6.6(5.4,8.1)^{b}$ & $6.2(5.5,7.2)$ & $6.1(5.2,7.2)$ \\
\hline Proteins, E\% & $16.6(14.8,18.5)$ & $17.1(15.3,19.0)^{a}$ & $15.9(14.6,18.0)$ & $15.4(13.7,17.3)^{\mathrm{a}}$ & $16.0(14.5,18.0)$ & $16.7(15.0,19.3)$ \\
\hline Proteins per kg & $1.07(0.88,1.29)$ & $1.02(0.85,1.20)^{b}$ & $0.85(0.69,1.00)^{c}$ & $0.77(0.65,0.96)^{c}$ & $0.96(0.80,1.13)^{a}$ & $0.97(0.83,1.16)^{a}$ \\
\hline Alcohol, E\% & $0.9(0,3.1)$ & $1.0(0,3.1)$ & $0(0,1.9)^{\mathrm{a}}$ & $0(0,0.9)^{b}$ & $0(0,1.0)^{b}$ & $0.4(0,1.9)$ \\
\hline Sucrose, E\% & $7.1(5.0,9.8)$ & $6.5(4.6,9.1)^{\mathrm{a}}$ & $6.0(4.8,9.1)$ & $5.9(4.7,7.8)^{a}$ & $7.2(6.0,10.3)$ & $6.6(3.5,8.7)^{\mathrm{a}}$ \\
\hline Fibre, g/MJ & $2.7(2.2,3.3)$ & $3.0(2.4,3.6)^{c}$ & $2.9(2.4,3.8)^{c}$ & $2.7(2.2,3.2)$ & $2.2(1.9,2.6)^{a}$ & $2.9(2.5,3.6)^{b}$ \\
\hline $\mathrm{Na}, \mathrm{g}$ & $2.8(2.4,3.3)$ & $2.7(2.2,3.1)^{c}$ & $2.6(2.0,3.1)^{c}$ & $2.5(2.2,2.9)^{b}$ & $2.7(2.2,3.3)$ & $2.7(2.2,3.2)^{a}$ \\
\hline $\mathrm{K}, \mathrm{g}$ & $4.0(3.3,4.7)$ & $3.9(3.4,4.6)$ & $3.8(3.1,4.6)^{b}$ & $3.0(2.4,3.7)^{\mathrm{a}}$ & $2.9(2.6,3.3)^{c}$ & $3.4(3.1,4.1)^{c}$ \\
\hline $\mathrm{Ca}, \mathrm{g}$ & $1.1(0.8,1.4)$ & $1.0(0.8,1.3)^{c}$ & $0.8(0.6,1.1)^{c}$ & $0.5(0.4,0.7)^{a}$ & $0.7(0.5,1.0)^{c}$ & $0.9(0.8,1.1)^{c}$ \\
\hline$P, g$ & $1.6(1.3,1.9)$ & $1.5(1.3,1.8)^{b}$ & $1.3(1.0,1.7)^{\mathrm{C}}$ & $1.1(0.9,1.2)^{a}$ & $1.2(1.0,1.4)^{\mathrm{c}}$ & $1.4(1.2,1.7)^{\mathrm{C}}$ \\
\hline
\end{tabular}

Data are presented as median (interquartile range) or mean \pm standard deviation. In these variables, comparisons between the highest eGFR group and the other groups were done with MannWhitney $U$ test, and independent samples t-test, respectively. eGFR, estimated glomerular filtration rate (unit ml/min/1.73 $\mathrm{m}^{2}$ ); E\%, percentage of energy intake; SAFA, saturated fatty acids; MUFA,

monounsaturated fatty acids; PUFA, polyunsaturated fatty acids; Na, sodium; K, potassium; Ca, calcium; P, phosphorus.

${ }^{a} p<0.05$.

b $p<0.01$.

${ }^{c} p<0.001$. 
ferences in the types of breads were more pronounced, however. A significantly lower frequency of rye bread consumption was observed in those with eGFR ${ }^{<30}$ and dialysis, but reached the level of those in the index group in kidney transplant recipients. In contrast, the frequencies of wheat bread consumption were higher in eGFR ${ }^{<30}$ and dialysis. Compared to the index group, there was a higher frequency of participants aiming at reducing salt consumption in all other groups. The use of probiotic supplements did not differ between the groups. Instead, the use of vitamin or mineral supplements was less frequent in those with $\mathrm{eGFR}^{\geq 30-59}$, $\mathrm{eGFR}^{<30}$, and in kidney transplant recipients. The frequencies of individuals reporting having received dietary advice from a dietitian was higher in all groups except in eGFR ${ }^{\geq 60-89}$, as compared to those with normal or high eGFR. Compared to the index group, the rate of self-reported compliance with dietary recommendations was higher in all groups. Special diet adherence was more frequent in all groups from eGFR ${ }^{\geq 30-59}$ onwards.

The differences in food consumption between individuals on dialysis and kidney transplant recipients was also investigated. Liquid milk product consumption $(p<0.001)$ and the number of bread slices eaten $(p=0.019)$ were higher in kidney transplant recipients. Moreover, the frequency of reporting rye bread consumption $(p<0.001)$ was higher, while that of wheat bread $(p<0.001)$ was lower in kidney transplant recipients. Finally, the frequency of special diet adherence $(p<0.001)$ was lower in those with kidney transplantation.

\subsection{Dietary patterns}

Seven dietary patterns were generated in the factor analysis (Supplementary Table 1). Compared to the index group, significantly lower High-fat cheese pattern scores were observed in those on dialysis (Table 3). Fish and vegetable pattern scores were higher in eGFR ${ }^{\geq 30-59}$ than in those with normal or high eGFR. In all groups were the Sweet pattern scores lower compared with the index group. However this difference did not reach statistical significance in the dialysis group. Those with eGFR ${ }^{260-89}$ had higher, and those on dialysis had lower Healthy snack pattern scores. In eGFR ${ }^{<30}$, Meat and potatos pattern scores were higher compared to the index group. Instead, the pattern scores of Pasta, rice and poultry were lower in $\mathrm{eGFR}^{\geq 60-89}$, eGFR ${ }^{\geq 30-59}$, and kidney transplant recipients.

Of the diet patterns, compared to the individuals on dialysis, kidney transplant recipients had higher scores in Fish and vegetables $(p=0.031)$, and Healthy snack $(p<0.001)$. Instead, the scores of Pasta, rice and poultry $(p=0.002)$ were lower in the kidney transplant recipients.

\subsection{Energy and nutrient intake}

In all, 1399 participants ( $41.7 \%$ men, age $48.9 \pm 13.7$ years) completed food records with plausible reported energy intake. All groups reported lower total energy intake compared to the index group (Table 4). Carbohydrate intake was comparable between the groups, but those on dialysis reported higher energy intake from fats. Compared to the index group, higher and lower energy intake from proteins was observed in eGFR ${ }^{\geq 60-89}$ and eGFR $^{<30}$, respectively. Instead, protein intake in $\mathrm{g} / \mathrm{kg}$ were, in all groups, lower than that in the index group. Lower sucrose intake was observed in eGFR ${ }^{\geq 60-89}$, eGFR ${ }^{<30}$, and kidney transplant recipients. Compared to the index group, those with eGFR ${ }^{\geq 60-89}$, eGFR ${ }^{\geq 30-59}$, and kidney transplant had higher, while those on dialysis lower fibre intake. The intakes of sodium, potassium, calcium, and phosphorus tended to be lower in all groups compared to the index group.

Compared to the individuals on dialysis, the percentage of energy from total fats $(p=0.036)$, saturated fatty acids $(p=0.038)$, and monounsaturated fatty acids $(p=0.017)$ were lower in kidney transplant recipients. In contrast, the intakes of fibre $(p<0.001)$, potassium $(p<0.001)$, calcium $(p<0.001)$, and phosphorus $(p<0.001)$ were higher in kidney transplanted individuals.

\section{Discussion}

To the best of our knowledge, this is the first study to investigate dietary intake at the levels of foods, dietary patterns, and nutrients in T1D at different stages of renal disease. We observed significant differences at all levels of dietary intake between those with normal renal function and those with different levels of renal deterioration. Based on higher selfreported compliance, greater aims at reducing salt intake, and lower scores in the diet pattern related to the intake of sweet food items, it seems that there was a general trend towards healthier food choices upon advancing kidney disease, despite obvious dietary restrictions. Indeed, many of the observed differences, such as reduction in the consumption of milk products and rye bread, higher frequencies of wheat bread consumption and special diet adherence, and reduced protein intake are likely signs of individuals following a diet plan aimed at delaying or managing kidney failure. A number of differences were also observed between individuals on dialysis and kidney transplant recipients. Amongst others, differences in milk product and bread consumption were seen. Importantly, a 36 percentage-point reduction in adherence to special diets likely represents the liberation from dietary restrictions imposed upon dialysis. Dietary liberation was also observed at micronutrient level, as kidney transplant recipients reported higher fibre, potassium, calcium, and phosphorus intakes.

Of importance, dietary intake did not only differ between individuals with intact kidney function and in more advanced kidney failure, but many of the differences were obvious already at the earlier stages of renal deterioration. The reasons or consequences of these differences are not known and, as most of the previous studies have quite exclusively focused on the dietary intake when renal deterioration has already progressed into end-stage renal disease, comparing these observations with the previous ones is challenging. In one study assessing dietary intake in haemodialysis, lower intakes of total energy, protein, and calcium were reported in individuals with diabetes compared to those without [18]. Another study of haemodialysis reported higher intakes of total energy, protein, fat, and fibre in men with diabetes compared to men without [19]. Moreover, compared to women 
without, women with diabetes had lower fat and carbohydrate but higher fibre intakes. In both sexes, individuals with diabetes reported lower sodium but higher potassium and phosphorus intakes compared to their non-diabetic counterparts. We found one study in T1D reporting dietary intake before and after islet transplantation [20]. In that study, no differences were observed in the intakes of energy or fibre, or proportions of energy derived from carbohydrates, fats and proteins before and after transplantation. Instead, significant reduction in phosphorus intake following the transplantation was reported.

While the number of studies on dietary intake at various stages of renal disease is limited, a larger number of papers have been published on the association between diet and kidney failure progression. Of the macronutrients, protein has probably gained the most interest and a number of metaanalyses have been published on the subject. The authors of a Cochrane meta-analysis including 7 randomized controlled trials concluded that protein restriction in T1D resulted in non-significant reduction in the GFR decline [21]. In contrast, the authors of a more recent meta-analysis concluded that protein restriction slowed CKD progression in individuals without diabetes and in T1D, but not in type 2 [22]. Yet in another meta-analysis of 13 randomized controlled trials including 779 participants with either type 1 or type 2 diabetes, low-protein diet was associated with significant improvement in GFR, an effect that was consistent across the diabetes subgroups [23]. However, this improvement was evident only when compliance to the diet was fair. Importantly, not only poor adherence to the prescribed diet, but also short duration of the trials, may mask the true effects of protein restriction. Partly due to the mixed results, it has also been suggested that the potential kidney sparing effect may not be directly related to the reduced protein intake, but may be confounded by the concomitant reduction in the salt or saturated fatty acid intakes [24]. While it remains unclear whether protein restriction slows the progression of diabetic kidney disease, there may be value in reduced-protein diets in patients with overt kidney failure. In these patients, however, the potential for malnutrition and hypoalbuminaemia, related to low-protein diet, need to be acknowledged [24]. Of note, the proportion of underweight participants amongst those on dialysis and with kidney transplant was also observed in the current study. At the same time, we observed higher waist-to-hip ratios in these two groups, suggesting that instead of losing fat mass these individuals had rather lost lean tissue.

The role of other dietary macronutrients in the diabetic kidney disease has been less investigated. One case report of a type 2 diabetes patient reducing his daily carbohydrate intake to $80-90 \mathrm{~g}$, equalling to $20 \%$ of energy from carbohydrates, showed both improved glycaemic control and reversal of a 6-year long decline in renal function [25]. The authors of a paper describing a 12-month intervention with moderate lowcarbohydrate diet (38 E\% carbohydrates), reported remarkable reduction in the urinary albumin excretion in individuals with type 2 diabetes and baseline microalbuminuria [26]. Studies in T1D are, again, scarce but in one such study 11 adult individuals adhering to a low-carbohydrate high-fat ketogenic diet (6 E\% carbohydrates, 65 E\% fats) displayed no or little evidence of renal dysfunction [27].

Importantly, foods and nutrients are not consumed in isolation, but cluster to form dietary patterns. These dietary patterns offer an attractive avenue for studying the association between diet and health. A Western dietary pattern, for example, characterized by high intakes of red meat, refined grains, and high-sugar drinks and desserts, has typically been associated with inflammation, impairment of renal vascular function, and decrease in kidney function [28,29]. A prudent diet, instead, defined as closer adherence with dietary recommendations, has proven beneficial for the kidneys as individuals with type 2 diabetes in the highest tertile of healthy eating index, compared to those in the lowest, showed reduced incidence or progression of CKD [6]. The Dietary Approaches to Stop Hypertension (DASH) diet pattern is another example of a prudent dietary pattern, and is characterized by high intakes of fruits, vegetables, nuts, legumes, low-fat dairy products, and whole grains, but low intakes of sodium, sweetened beverages, and red and processed meats. In the Nurses' Health Study, individuals in the top quartile of the DASH-diet score, compared to those in the bottom quartile, had significantly decreased risk of rapid eGFR decline [29]. Finally, of the factor analysis-derived dietary patterns, one with abundant intake of fish and vegetables was associated with better kidney function in a cross-sectional study of individuals with type 2 diabetes [30].

The use of validated methods to study dietary intake and a well-defined study population are notable strengths of this study. There are, however, also important limitations that need to be addressed. Despite a fair number of participants in this study, the numbers of individuals in some of the groups of renal status were quite modest. Also, individuals taking part in health-related studies may be different from the ones that decline to participate. It is likely, for example, that individuals with greater interest in health and diet, in particular, are over-represented in the current sample. Taken together, these could reduce the generalizability of the results to the T1D population at large. As this is a cross-sectional study, inferences about the role of diet in the progression of renal disease cannot be made. Instead, our aim was rather to evaluate the current diet of individuals in different stages of renal disease. This study will serve as a baseline for future analyses where the longitudinal associations will be assessed. The use of self-reported methods for measuring dietary intake is a common practice in epidemiological studies. However, these methods are prone to misreporting, and it has been suggested that self-report methods should not, for example, be used to assess absolute energy intake [31]. Due to social desirability, there may also be a tendency for participants to over- and under-report food items that are considered healthy and unhealthy, respectively. In the current study we used two separate methods, with partly different sources of error, to study dietary intake. The potential for misreporting remains, however, and it is not known whether renal status is associated with the level of misreporting.

In conclusion, results from the current study suggest that dietary intake differ based on the level of renal status. Differences were observed, not only at advanced kidney disease, 
but already at earlier stages of eGFR decline. The observed differences reflected the recommendations typically imposed to individuals with $\mathrm{T} 1 \mathrm{D}$ with deteriorating renal function. While receiving a kidney transplant marks the end of a more restrictive diet of pre-dialysis and dialysis periods, the diets of the kidney transplant recipients, with many respect, still differed from those with intact renal function.

\section{Acknowledgements}

The skilled technical assistance of Anna Sandelin, Mira Korolainen, and Jaana Tuomikangas is gratefully acknowledged. The authors also acknowledge all the physicians and nurses at each centre participating in the collection of patients (Supplementary List of participating FinnDiane Study Centers).

\section{Declaration of Competing Interest}

Professor Per-Henrik Groop has received grants from Eli Lilly and Roche, is an advisory board member for AbbVie, Astellas, Astra Zeneca, Boehringer-Ingelheim, Cebix, Eli Lilly, Janssen, MSD, Medscape, Mundipharma, Novartis, Novo Nordisk, and Sanofi. He has received lecture honoraria from Astra Zeneca, Boehringer-Ingelheim, Eli Lilly, Elo Water, Genzyme, MSD, Novartis, Novo Nordisk, PeerVoice, and Sanofi. All other authors declare no conflicts of interest.

\section{Authors' contributions}

All authors participated in designing the study. AJA completed the analyses, and drafted the manuscript. All authors helped to interpret the data, revised the manuscript, provided intellectual content to the work, and gave their final approval to the paper to be published.

\section{Funding}

This study was supported by grants from Academy of Finland (grant number 316664), Novo Nordisk Foundation (\#NNF14SA0003), Signe and Ane Gyllenberg Foundation, Folkhälsan Research Foundation, Helsinki University Central Hospital Research Funds, Wilhelm and Else Stockmann Foundation, Liv och Hälsa Society, Päivikki and Sakari Sohlberg Foundation. Funding agencies did not contribute to the study design, conduct of the study, data analysis, interpretation of the findings, writing of the manuscript, or in the decision to submit the manuscript for publication.

\section{Appendix A. Supplementary material}

Supplementary data to this article can be found online at https://doi.org/10.1016/j.diabres.2019.06.016.

\section{R E F E R E N C E S}

[1] Levey AS, Coresh J. Chronic kidney disease. Lancet 2012;379:165-80.
[2] Ng JK, Li PK. Chronic kidney disease epidemic: how do we deal with it? Nephrology (Carlton) 2018;23(Suppl 4):116-20.

[3] Hovind P, Tarnow L, Rossing K, Rossing P, Eising S, Larsen N, et al. Decreasing incidence of severe diabetic microangiopathy in type 1 diabetes. Diabetes Care 2003;26:1258-64.

[4] Groop PH, Thomas MC, Moran JL, Wadén J, Thorn LM, Mäkinen VP, et al. The presence and severity of chronic kidney disease predicts all-cause mortality in type 1 diabetes. Diabetes 2009;58:1651-8.

[5] Onyenwenyi C, Ricardo AC. Impact of lifestyle modification on diabetic kidney disease. Curr Diab Rep 2015;15. 60-0150632-3.

[6] Dunkler D, Kohl M, Teo KK, Heinze G, Dehghan M, Clase CM, et al. Dietary risk factors for incidence or progression of chronic kidney disease in individuals with type 2 diabetes in the European Union. Nephrol Dial Transplant 2015;30(Suppl 4). iv76-85.

[7] Satirapoj B, Adler SG. Comprehensive approach to diabetic nephropathy. Kidney Res Clin Pract 2014;33:121-31.

[8] Evert AB, Boucher JL, Cypress M, Dunbar SA, Franz MJ, MayerDavis EJ, et al. Nutrition therapy recommendations for the management of adults with diabetes. Diabetes Care 2014;37 (Suppl 1):S120-43.

[9] American Diabetes Association. 4. Lifestyle management: Standards of medical care in diabetes-2018. Diabetes Care 2018;41(Suppl 1):S38-50.

[10] Willingham F. The dietary management of patients with diabetes and renal disease: challenges and practicalities. J Ren Care 2012;38(Suppl 1):S40-51.

[11] Fernandes AS, Ramos CI, Nerbass FB, Cuppari L. Diet quality of chronic kidney disease patients and the impact of nutritional counseling. J Ren Nutr 2018;28:403-10.

[12] Naylor HL, Jackson H, Walker GH, Macafee S, Magee K, Hooper L, et al. British dietetic association evidence-based guidelines for the protein requirements of adults undergoing maintenance haemodialysis or peritoneal dialysis. J Hum Nutr Diet 2013;26:315-28.

[13] Ahola AJ, Thorn LM, Saraheimo M, Forsblom C, Groop PH, Finndiane Study Group. Depression is associated with the metabolic syndrome among patients with type 1 diabetes. Ann Med 2010;42:495-501.

[14] Levey AS, Stevens LA, Schmid CH, Zhang YL, Castro AF, Feldman HI, et al. A new equation to estimate glomerular filtration rate. Ann Intern Med 2009;150:604-12.

[15] KDIGO 2012 Clinical Practice Guideline for the Evaluation and Management of Chronic Kidey Disease. http://www.kdigo. org/clinical_practice_guidelines/pdf/CKD/KDIGO_2012_CKD_ GL.pdf [accessed February 2019].

[16] Ahola AJ, Mikkilä V, Mäkimattila S, Forsblom C, Freese R, Groop PH. Energy and nutrient intakes and adherence to dietary guidelines among Finnish adults with type 1 diabetes. Ann Med 2012;44:73-81.

[17] Ahola AJ, Lassenius MI, Forsblom C, Harjutsalo V, Lehto M. Groop PH. Dietary patterns reflecting healthy food choices are associated with lower serum LPS activity. Sci Rep 2017;7 6511-017-06885-7.

[18] Bataille S, Landrier JF, Astier J, Cado S, Sallette J, Giaime P, et al. Haemodialysis patients with diabetes eat less than those without: a plea for a permissive diet. Nephrology (Carlton) 2017;22:712-9.

[19] Bogacka A, Sobczak-Czynsz A, Kucharska E, Madaj M, Stucka $\mathrm{K}$. Analysis of nutrition and nutritional status of haemodialysis patients. Rocz Panstw Zakl Hig 2018;69:165-74.

[20] Poggioli R, Enfield G, Messinger S, Faradji RN, Tharavanij T, Pisani L, et al. Nutritional status and behavior in subjects 
with type 1 diabetes, before and after islet transplantation. Transplantation 2008;85:501-6.

[21] Robertson L, Waugh N, Robertson A. Protein restriction for diabetic renal disease. Cochrane Database Syst Rev 2007;4: CD002181.

[22] Rughooputh MS, Zeng R, Yao Y. Protein diet restriction slows chronic kidney disease progression in non-diabetic and in type 1 diabetic patients, but not in type 2 diabetic patients: a meta-analysis of randomized controlled trials using glomerular filtration rate as a surrogate. PLoS One 2015;10: e0145505.

[23] Nezu U, Kamiyama H, Kondo Y, Sakuma M, Morimoto T, Ueda S. Effect of low-protein diet on kidney function in diabetic nephropathy: meta-analysis of randomised controlled trials. BMJ Open 2013;3. https://doi.org/10.1136/bmjopen-2013002934.

[24] Hamdy O, Horton ES. Protein content in diabetes nutrition plan. Curr Diab Rep 2011;11:111-9.

[25] Nielsen JV, Westerlund P, Bygren P. A low-carbohydrate diet may prevent end-stage renal failure in type 2 diabetes. A case report. Nutr Metab (Lond) 2006;3. 23-7075-3-23.

[26] Haimoto H, Sasakabe T, Umegaki H, Wakai K. Reduction in urinary albumin excretion with a moderate low- carbohydrate diet in patients with type 2 diabetes: a 12month intervention. Diabetes Metab Syndr Obes 2012;5:283-91.

[27] Leow ZZX, Guelfi KJ, Davis EA, Jones TW, Fournier PA. The glycaemic benefits of a very-low-carbohydrate ketogenic diet in adults with type 1 diabetes mellitus may be opposed by increased hypoglycaemia risk and dyslipidaemia. Diabet Med 2018. https://doi.org/10.1111/dme.13663.

[28] Odermatt A. The Western-style diet: a major risk factor for impaired kidney function and chronic kidney disease. Am J Physiol Renal Physiol 2011;301:F919-31.

[29] Lin J, Fung TT, Hu FB, Curhan GC. Association of dietary patterns with albuminuria and kidney function decline in older white women: A subgroup analysis from the nurses' health study. Am J Kidney Dis 2011;57:245-54.

[30] Chung HF, Hsu CC, Mamun AA, Long KZ, Huang YF, Shin SJ, et al. Dietary patterns, dietary biomarkers, and kidney disease in patients with type 2 diabetes: a repeated-measure study in Taiwan. Asia Pac J Clin Nutr 2018;27:366-74.

[31] Subar AF, Freedman LS, Tooze JA, Kirkpatrick SI, Boushey C, Neuhouser ML, et al. Addressing current criticism regarding the value of self-report dietary data. J Nutr 2015;145:2639-45. 